\title{
Isolated Hyperthermic Hepatic Chemopertusion in Patients with Colorectal Cancer and solated Non-Resectable Liver Metastases
}

DOI: $10.17691 /$ stm2018.10.3.19

Received November 6, 2017

A.I. Babich, MD, PhD, Deputy Surgeon-in-Chief;

V.M. Unguryan, MD, PhD, Physician-in-Chief;

Yu.A. Pobedintseva, MD, Deputy Physician-in-Chief in Charge of Anesthesiology and Resuscitation;

V.A. Kudlachev, MD, Deputy Physician-in-Chief in Charge of Clinical Service;

E.A. Kruglov, MD, Oncologist

Kostroma Oncology Center, 38 Malyshkovskaya St., Kostroma, 156011, Russia

The aim of the study was to evaluate the clinical efficacy of isolated hyperthermic hepatic chemoperfusion (IHHC) in patients with colorectal cancer and isolated non-resectable liver metastasis $\left(\mathrm{M}_{1 \mathrm{c}}\right)$ resistant to systemic polychemotherapy (PCT).

Materials and Methods. The study included 6 patients with colorectal cancer with isolated non-resectable liver metastatic lesions resistant to systemic PCT. The average age of patients was $59.3 \pm 3.4$ years. Before the IHHC, all patients received 2 cycles or more of PCT that failed to prevent an increase in metastatic foci in the liver. The IHHC was performed using an artificial circulation device; the chemotherapeutic agents (melphalan in combination with TNF- $\alpha$ ) were administered through the gastroduodenal artery. Blood from the retro-hepatic segment of the inferior vena cava was collected after the liver vessels were completely isolated and a parallel circulation shunt was established for returning the blood into the superior vena cava from the inferior vena cava and the portal vein

Results. In 3 patients with follow-up examinations at 3 and 6 months, a complete response (according to the mRECIST scores) was noted; in the other 3 patients, there was a partial response to the treatment. In patients with a complete response, the use of IHHC resulted in complete tumor pathomorphism confirmed by histology and spiral CT at 3 and 6 months after the procedure.

Conclusion. IHHC with melphalan and TNF- $\alpha$ can be used for regional chemotherapy in patients with isolated non-resectable metastases resistant to systemic chemotherapy.

Key words: isolated perfusion; isolated liver chemoperfusion; colorectal cancer; regional chemotherapy; metastases to the liver; melphalan; tumor necrosis factor.

\section{Introduction}

Despite the achievements of modern surgery and oncology, treatment of isolated metastatic liver lesions in colorectal cancer presents a significant clinical challenge $[1,2]$. This issue needs to be urgently addressed because in $80 \%$ of patients (at the moment liver metastasis is confirmed), the lesion is already bilobar, which rules out performing any radical resection [3]. Biological characteristics of colorectal cancer suggest that the available adjuvant chemotherapy is of limited efficacy in such patients: the response rate to secondline chemotherapy is less than $25 \%$, and the survival period on the second-line chemotherapy is less than 12 months [4-6].

The successful experience with regional chemotherapy in patients with isolated metastatic liver disease is mainly due to the use of intra-arterial chemoembolization of liver metastases via the hepatic artery [7]. However, this route is not suitable for chemotherapy with melphalan and TNF- $\alpha$ because of their high systemic toxicity $[8,9]$. In addition, during intraarterial chemoembolization, it is not possible to maintain the level of hyperthermia required for the efficient antitumor therapy [10]. The isolated hyperthermic hepatic chemoperfusion (IHHC) can produce local hyperthermia and bring a chemotherapeutic agent to the target, preventing it from entering the systemic circulation $[6,10,11]$. IHHC was reported to cause a significant clinical improvement and even a complete tumor regression $[4,8,9]$.

For the first time, the technique of IHHC was presented Ausman [12], who conducted a series of experimental studies on dogs with the subsequent introduction of the technique into clinical practice. Then, over 40 years, an interest in the technique resumed for a while and then dropped again, mainly due to the complexity of the procedure and high perioperative mortality $[5,10]$. By now, around $500 \mathrm{IHHC}$ procedures have been completed worldwide; most of them

Corresponding author: Alexander I. Babich, e-mail: gd@medex-oms.ru 
performed by Dr. Alexander in the US [4] and Dr. van lersel in Europe [2]. Unfortunately, to date, there is no published data on completed IHHC procedures in Russia.

The aim of the study was to test the feasibility and clinical efficacy of isolated hyperthermic hepatic chemoperfusion with melphalan and TNF- $\alpha$ for the treatment of isolated bilobar metastatic lesions in patients with colorectal cancer failed to respond to systemic chemotherapy.

\section{Material and Methods}

The study was carried out in accordance with the requirements of the Helsinki Declaration (2013). The authors received a written consent for IHHC from all patients.

Six patients ( 4 males, 2 females) participated in the study. The average age of the patients was $59.3 \pm 3.4$ years (45-70 years). Isolated bilobar metastatic liver lesions $\left(M_{1 c}\right)$ were confirmed in all patients; in three patients, the liver metastases were diagnosed simultaneously with the primary diagnosis, and in three others - during the post-treatment follow-up. At the time of the detection, the liver metastases were diagnosed as unresectable in all patients; the lesions were localized in both the right and left lobes; therefore, it was impossible to perform a radical resection and leave sufficient tissue for proper liver function. The location of the primary tumor in 3 patients was the rectum, and in other 3 - the colon. The histological type of the tumor in all cases was adenocarcinoma; in 2 cases - moderately differentiated (G2) and in 4 cases - poorly differentiated (G3).

The large intestine tumors were removed using the standard resection procedures (in the rectum - total

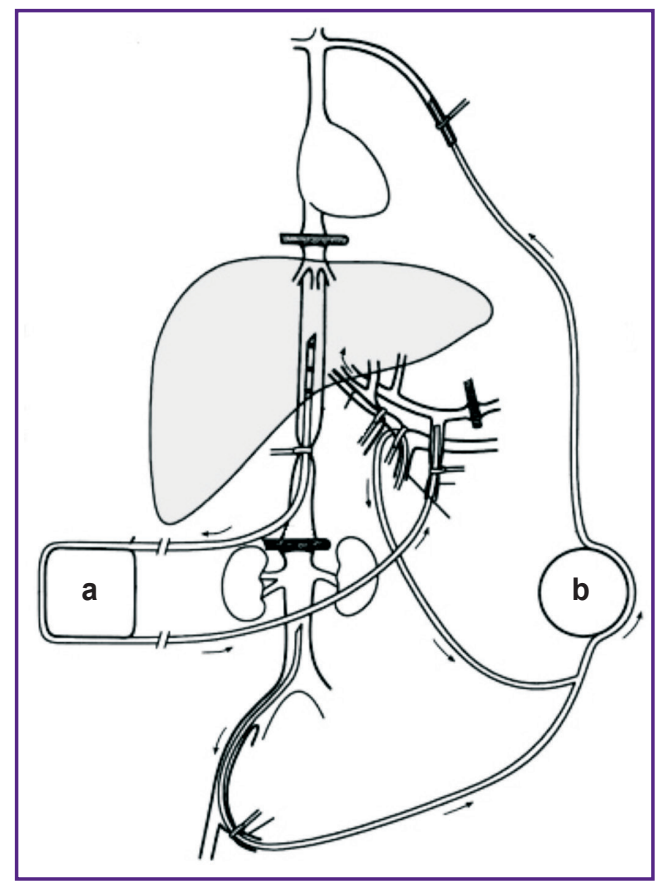

mesorectal excision with rectum resection, in the colontotal mesorectal excision with partial colon resection). Before the IHHC, all patients underwent two or more cycles of polychemotherapy; despite that, an increase in the size of secondary foci in the liver was found. Given the poor efficacy of systemic polychemotherapy, the patients were scheduled for IHHC.

Isolated hyperthermic liver perfusion was performed according to our original modification (priority certificate No.2017118306 dated May 25, 2017). It included a step of vascular isolation of the liver followed by creation of two closed loops of artificial circulation:

in the $1^{\text {st }}$, the main one (blood circulation through the liver), a solution was pumped into the liver through a cannula installed in the gastroduodenal artery, and the blood coming from the liver entered a cannula installed in the retro-hepatic segment of the inferior vena cava;

in the $2^{\text {nd }}$ shunt (provides blood return from the lower extremities, kidneys, and the intestines to the heart), the blood was withdrawn through a cannula installed in the sub-hepatic segment of the inferior vena cava (from the kidneys and lower limbs), and through a cannula installed in the portal vein; within this route, the blood returned into the internal jugular vein via a puncturemounted introducer with a diameter of $3 / 16$.

Before the cannulation, heparin was administered to provide for the activated clotting time (ACT) of $400 \mathrm{~s}$. The main artificial circuit was filled with a mixture containing: $300 \mathrm{ml}$ of erythrocyte mass, $700 \mathrm{ml}$ of saline and chemotherapeutic agents - melphalan $(1.5 \mathrm{mg} / \mathrm{kg}$ body mass) together with $1 \mathrm{mg}$ TNF- $\alpha$. This mixture was pumped through an oxygenator and a heat exchanger before entering the gastroduodenal artery.

The scheme of the main perfusion circuit and the vein-venous shunt is shown in Figure 1.
Figure 1. Scheme of the main perfusion circuit and the vein-venous shunt:

(a) the reservoir for pooling the blood from the retro-hepatic segment of the inferior vena cava and then pushing it to the gastroduodenal artery (the main perfusion circuit); (b) the reservoir for pooling the blood from the inferior vena cava and the portal vein and then pushing it to the superior vena cava (vein-venous shunt); the arrows indicate the blood flow directions 
The major parameters of this artificial circulation were as follows: the volume velocity of perfusion along the main circuit - 800-1100 $\mathrm{ml} / \mathrm{min}$; the pressure in the arterial cannula - 120-180 mm Hg; under hyperoxia, partial oxygen tension in the feeding mixture was $>150 \mathrm{~mm} \mathrm{Hg}$; in hyperthermia, temperature of $40^{\circ} \mathrm{C}$ was maintained; the volume velocity of the shunt flow 800-1200 $\mathrm{ml} / \mathrm{min}$; duration of isolated liver perfusion $60 \mathrm{~min}$. After the end of the IHHC session, the perfusate was washed out from the liver using $1500 \mathrm{ml}$ saline and $500 \mathrm{ml}$ gelofusine. Then, the cannulas were removed and the vascular defects were sutured; heparin was neutralized with a solution of protamine sulfate (10 $\mathrm{U}$ of protamine per 1000 units of heparin); cholecystectomy was performed, and the laparotomy wound was sutured layer-by-layer.

The response to the treatment was determined by spiral CT (the mRECIST score), as well as by the level of tumor markers CA 19-9 and CEA (cancerembryonic antigen). For the morphological study of liver metastases, an incisional biopsy of the tumor tissue was performed immediately after the laparotomy and one hour after the IHHC. The tissue samples were sent for histological and immunohistochemical examination [4]. Immunohistochemical studies were performed to measure the level of Ki-67.

Given a small number of clinical observations, the data was processed using nonparametric methods (the Wilcoxon test and the Mann-Whitney test).

\section{Results and Discussion}

The duration of surgical intervention averaged at $267 \pm 43 \mathrm{~min}$, the intraoperative blood loss was $475 \pm 95 \mathrm{ml}$. During the operation, no blood transfusions were performed. There were no lethal outcomes during the patients' hospital stay. In the postoperative period, all patients showed a transient increase in the levels of transaminases: the ALT and AST elevated up to $270 \mathrm{mmol} / \mathrm{L}$ within $24 \mathrm{~h}$ after the operation and then returned to a normal range by day 7 in all patients. The total bilirubin did not exceed $15 \mathrm{mmol} / \mathrm{L}$. During the postoperative period, one patient developed acute renal failure; after three sessions of ultra-hemodiafiltration, a normal pace of diuresis restored and the parameters of nitrogen metabolism normalized. The duration of the patients' stay in the ICU was $4 \pm 1$ days, the total period of hospitalization $-13 \pm 3$ days.

The above data on the operation time and the intraoperative blood loss were comparable with the results of others [4]. The development of renal insufficiency in the postoperative period in one patient could be explained by rhabdomyolysis (the level of myoglobin in the blood reached $1000 \mu \mathrm{g} / \mathrm{L}$ ) as a result of hypoperfusion of skeletal muscles caused by vasospasm and the vasopressor support. Such a complication of the perfusion procedure was earlier reported by others [12], but the authors attributed it to rhabdomyolysis caused by a leakage of the chemotherapeutic drug. In the present study, there were no indications of melphalan or TNF-a leakage, since there were no manifestations of systemic toxicity (primarily suppression of hematopoiesis).

The immediate results of $\mathrm{IHHC}$ assessed by morphological data showed tumor necrosis and fibrosis in 3 patients (Figure 2).

The long-term results of IHHC assessed after 3 and 6 months indicated a complete response in 3 patients (Figure 3) and a partial response in three others.

In patients with a complete response to $\mathrm{IHHC}$, the oncomarkers (CA 19-9 and CEA) decreased to normal values; in patients with a partial response, the levels of oncomarkers were lower than those before IHHC but still above normal. In our study, the number of patients with complete tumor responses is higher (in the percentage
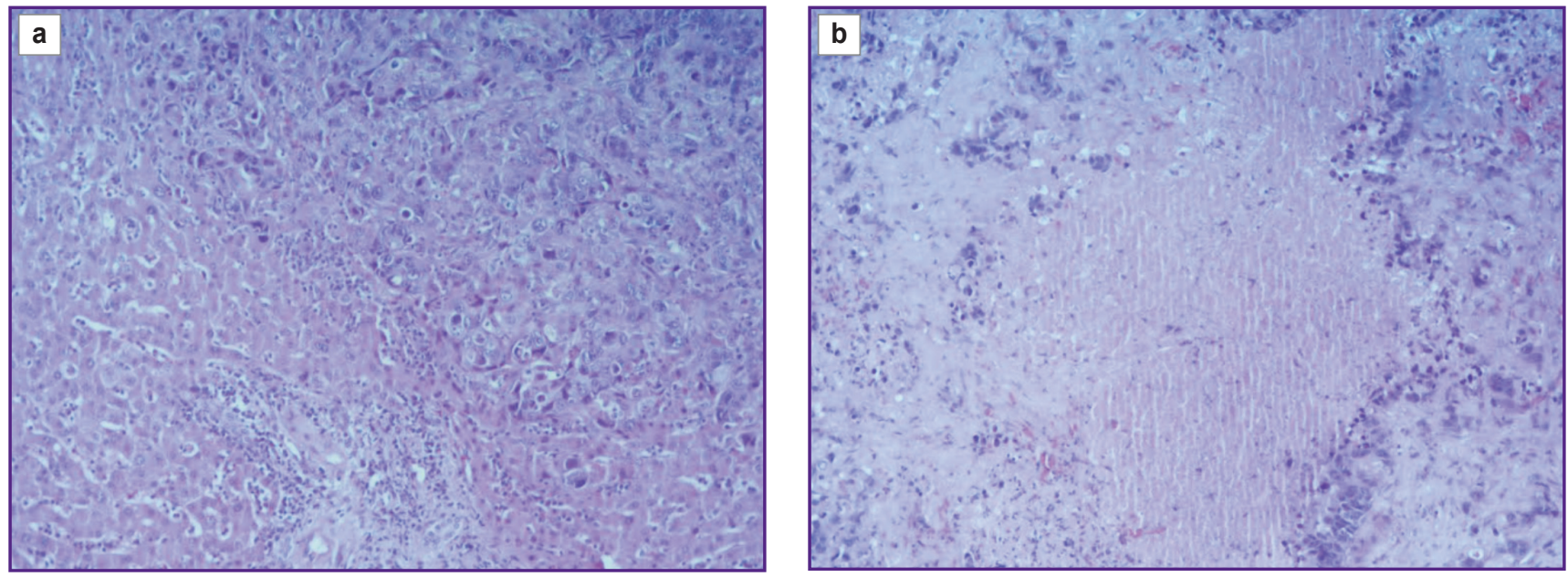

Figure 2. Histological examination of liver metastases:

(a) before isolated hyperthermic hepatic chemoperfusion, adenocarcinoma G3; (b) after isolated hyperthermic hepatic chemoperfusion, necrosis and fibrosis; staining with hematoxylin and eosin; $\times 10$ 


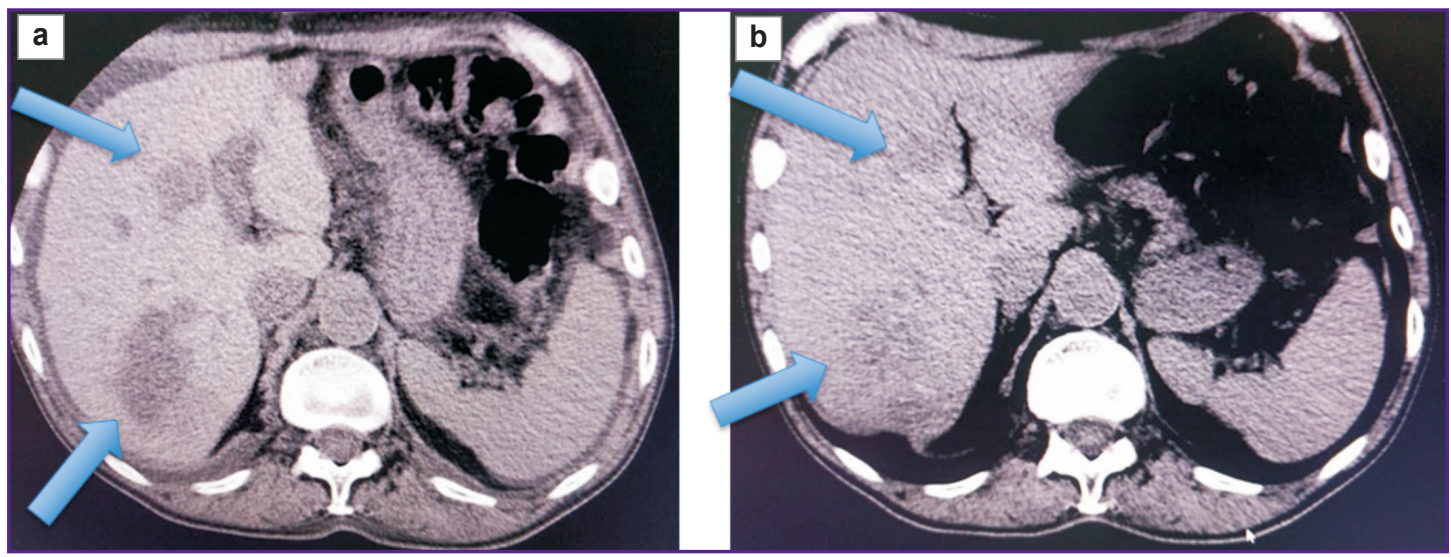

Figure 3. Complete tumor response to the isolated hyperthermic hepatic chemoperfusion evaluated with the MRECIST scale:

the arrows indicate metastases of colorectal cancer (a) and fibrosis in the area of metastases localization after isolated hyperthermic hepatic chemoperfusion (b)

terms) than that in the reports of other authors [1, 4]. This, of course, can be due to a small number of patients, which necessitates a continuation of the study on a larger group of patients.

Otherwise, our results are consistent with the data of others $[4,8,9]$.

\section{Conclusion}

Isolated hyperthermic liver chemoperfusion with melphalan and TNF- $\alpha$ can serve as an effective method of regional chemotherapy indicated in patients with isolated non-resectable metastatic liver lesions resistant to systemic chemotherapy.

Financial support. This work was funded by the authors.

Conflict of interest. The authors declare no conflicts of interest.

\section{References}

1. Leporrier J., Maurel J., Chiche L., Bara S., Segol P., Launoy G. A population-based study of the incidence, management and prognosis of hepatic metastases from colorectal cancer. Br J Surg 2006; 93(4): 465-474, https://doi. org/10.1002/bjs.5278.

2. van lersel L.B., Koopman M., van de Velde C.J., Mol L., van Persijn van Meerten E.L., Hartgrink H.H., Kuppen P.J., Vahrmeijer A.L., Nortier J.W., Tollenaar R.A., Punt C., Gelderblom H. Management of isolated nonresectable liver metastases in colorectal cancer patients: a case-control study of isolated hepatic perfusion with melphalan versus systemic chemotherapy. Ann Oncol 2010; 21(8): 1662-1667, https://doi. org/10.1093/annonc/mdp589.

3. Tzeng C.-W.D., Aloia T.A. Colorectal liver metastases. J Gastrointest Surg 2013; 17(1): 195-201, https://doi. org/10.1007/s11605-012-2022-3.
4. Alexander H.R. Jr., Libutti S.K., Bartlett D.L., Pingpank J.F., Kranda K., Helsabeck C., Beresnev T. Hepatic vascular isolation and perfusion for patients with progressive unresectable liver metastases from colorectal carcinoma refractory to previous systemic and regional chemotherapy. Cancer 2002; 95(4): 730-736, https://doi.org/10.1002/ cncr.10686.

5. Masi G., Vasile E., Loupakis F., Cupini S., Fornaro L., Baldi G., Salvatore L., Cremolini C., Stasi I., Brunetti I., Fabbri M.A., Puglisi M., Trenta P., Granetto C., Chiara S., Fioretto L., Allegrini G., Crinò L., Andreuccetti M., Falcone A. Randomized trial of two induction chemotherapy regimens in metastatic colorectal cancer: an updated analysis. J Natl Cancer Inst 2011; 103(1): 21-30, https://doi.org/10.1093/jnci/ djq456.

6. Rothenberg M.L., Cox J.V., Butts C., Navarro M., Bang Y.J., Goel R., Gollins S., Siu L.L., Laguerre S., Cunningham D. Capecitabine plus oxaliplatin (XELOX) versus 5 -fluorouracil/folinic acid plus oxaliplatin (FOLFOX-4) as second-line therapy in metastatic colorectal cancer: a randomized phase III noninferiority study. Ann Oncol 2008; 19(10): 1720-1726, https://doi.org/10.1093/annonc/mdn370.

7. Alexander H.R., Libutti S.K., Pingpank J.F., Bartlett D.L., Helsabeck C., Beresneva T. Isolated hepatic perfusion for the treatment of patients with colorectal cancer liver metastases after irinotecan-based therapy. Ann Sur Oncol 2005; 12(2): 138-144, https://doi.org/10.1245/aso.2005.05.003.

8. Rothbarth J., Pijl M.E., Vahrmeijer A.L., Hartgrink H.H., Tijl F.G., Kuppen P.J., Tollenaar R.A., van de Velde C.J. Isolated hepatic perfusion with high-dose melphalan for the treatment of colorectal metastasis confined to the live. Br J Surg 2003; 90(11): 1391-1397, https://doi.org/10.1002/bjs.4308.

9. Alexander H.R. Jr., Bartlett D.L., Libutti S.K., Pingpank J.F., Fraker D.L., Royal R., Steinberg S.M., Helsabeck C.B., Beresneva T.H. Analysis of factors associated with outcome in patients undergoing isolated hepatic perfusion for unresectable liver metastases from colorectal center. Ann Surg Oncol 2009; 16(7): 1852-1859, https://doi.org/10.1245/ s10434-009-0482-9.

10. Schwemmte K., Link K.H., Rieck B. Rationale and 
indications for perfusion in liver tumors: current data. World J Surg 1987; 11(4): 534-540, https://doi.org/10.1007/ bf01655820.

11. Aigner K., Walther H., Tonn J., Wenzl A., Hechtel R., Merker G., Schwemmle K. First experimental and clinical results of isolated liver perfusion with cytotoxics in metastases from colorectal primary. Recent Results in Cancer Research 1983; 86: 99-102, https://doi.org/10.1007/978-3-642-82025-0_18.

12. World Medical Association. World Medical Association Declaration of Helsinki: ethical principles for medical research involving human subjects. JAMA 2013; 310(20): 2191-2194, https://doi.org/10.1001/jama.2013.281053. 